\title{
Silica Aerogel: Synthesis and Applications
}

\author{
Jyoti L. Gurav, ${ }^{1}$ In-Keun Jung, ${ }^{1}$ Hyung-Ho Park, ${ }^{1}$ Eul Son Kang, ${ }^{2}$ and Digambar Y. Nadargi ${ }^{3}$ \\ ${ }^{1}$ Department of Materials Science and Engineering, Yonsei University, 120-749 Seoul, Republic of Korea \\ ${ }^{2}$ Agency for Defense Development, Daejeon 305-600, Republic of Korea \\ ${ }^{3}$ Empa, Swiss Federal Laboratories for Materials Science and Technology, Laboratory for Building Technologies, \\ 8600 Dubendorf, Switzerland
}

Correspondence should be addressed to Hyung-Ho Park, hhpark@yonsei.ac.kr

Received 26 January 2010; Revised 12 May 2010; Accepted 30 June 2010

Academic Editor: Ping Xiao

Copyright (c) 2010 Jyoti L. Gurav et al. This is an open access article distributed under the Creative Commons Attribution License, which permits unrestricted use, distribution, and reproduction in any medium, provided the original work is properly cited.

\begin{abstract}
Silica aerogels have drawn a lot of interest both in science and technology because of their low bulk density (up to 95\% of their volume is air), hydrophobicity, low thermal conductivity, high surface area, and optical transparency. Aerogels are synthesized from molecular precursors by sol-gel processing. Special drying techniques must be applied to replace the pore liquid with air while maintaining the solid network. Supercritical drying is most common; however, recently developed methods allow removal of the liquid at atmospheric pressure after chemical modification of the inner surface of the gels, leaving only a porous silica network filled with air. Therefore, by considering the surprising properties of aerogels, the present review addresses synthesis of silica aerogels by the sol-gel method, as well as drying techniques and applications in current industrial development and scientific research.
\end{abstract}

\section{Introduction}

The rapid development of sol-gel techniques during the past two decades has led to fast progress in the deliberate synthesis of porous materials. These techniques complement conventional procedures used for the preparation of amorphous solids or glasses, such as precipitation or impregnation methods followed by high-temperature treatments. Porous materials are of immense importance in various applications such as adsorption, sensing, and catalysis, owing to their high surface area, porosity, adjustable framework, and surface properties. Out of all known solid porous materials, aerogels are particularly known for their small pore size, large specific surface area, and best optical transmission. Further, among all aerogels, silica aerogels have become quite popular because they possess a wide variety of exceptional properties such as low thermal conductivity $(\sim 0.01 \mathrm{~W} / \mathrm{m} . \mathrm{K})$, high porosity ( $99 \%)$, high optical transmission $(99 \%)$ in the visible region, high specific surface area $\left(1000 \mathrm{~m}^{2} / \mathrm{g}\right)$, low dielectric constant $(\sim 1.0-2.0)$, low refractive index $(\sim 1.05)$, and low sound velocity $(100 \mathrm{~m} / \mathrm{s})[1-4]$.

Before exploring more details regarding silica aerogels, we first provide an overview of the history of aerogel inven- tion and its development. In the 1930s, Samuel Stephens Kistler first produced silica aerogels by formulating the idea of replacing the liquid phase by a gas with only a slight shrinkage of the gel. He prepared aerogels from many other materials, including alumina, tungsten oxide, ferric oxide, tin oxide, nickel tartarate, cellulose, cellulose nitrate, gelatin, agar, egg albumen, and rubber, which are out of scope of the discussion. Kistler's method involves tedious and timeconsuming procedures, and as such there was no follow-up interest in the field of aerogels until 1968 when rediscovery of aerogels took place by a team of researchers headed by Professor S. J. Teichner at the University Claude, Bernard, Lyon, France. They substantially simplified the procedure by carrying out the sol-gel transition in a solvent, which was then removed at supercritical conditions. The first Cerenkov radiation detector based on silica aerogels was developed in 1974 by Cantin et al. Since then, aerogels have been used or considered for use in laser experiments, sensors, thermal insulation, waste management, for molten metals, for optics and light guides, electronic devices, capacitors, imaging devices, catalysts, pesticides, and cosmic dust collection. More recently, several groups around the 
world began working in the field of silica aerogels for the various applications mentioned above. Strictly speaking, to understand silica aerogels, it is necessary to first understand sol-gel chemistry and related physicochemical aspects. In the following, we shall discuss sol-gel chemistry, synthetic strategy of silica aerogels, and some recent developments in applications of aerogels.

\section{Synthesis of Aerogel by Sol-Gel Process}

Sol-gel processing is a very popular and reliable methodology for the synthesis of materials, especially metal oxides with uniform, small particle sizes, and varied morphologies [510]. It involves the transition of a system from a liquid "sol" into a solid "gel" phase. The sol-gel process can ordinarily be divided into the following steps: forming a solution, gelation, aging, drying, and densification. A few of the important advantages of the sol-gel process are its simplicity, and the fact that it is an economic and effective means of producing high-quality materials. Sol-gel processing has found application in the production of high-quality glasses for optical components and fibers, thin film coatings, and fine oxide powders [11-15].

2.1. Sol-Gel Process. Sol-gel processing implies the synthesis of an inorganic network by a chemical reaction in solution at low temperatures or the formation of an amorphous network in opposition to crystallization from the solution. The most obvious feature of this reaction is the transition from a colloidal solution (liquid) into a di- or multiphase gel (solid) that led to the expression "sol-gel process".

The formation of uniform suspensions of colloidal particles can be understood by calculation of the sedimentation rates assuming that the particles are spherical so that Stokes' law can be applied. Sedimentation rate is

$$
\begin{aligned}
d x / d t & =\left[\left(4 \pi r^{3} / 3\right)\left(\rho^{\prime}-\rho\right) g\right] / 6 \pi r \eta \\
& =\left[2 r^{2}\left(\rho^{\prime}-\rho\right) g\right] / 9 \eta,
\end{aligned}
$$

where $\eta=$ viscosity of surrounding medium, $r=$ radius of colloid particle, $\rho^{\prime}=$ density of colloid particle material, and $\rho=$ density of surrounding material.

More clearly, a sol is a colloidal suspension of the solid particles in a liquid in which the dispersed phase is small $(1-1000 \mathrm{~nm})$. Therefore, the gravitational force is negligible and short-range forces, such as van der Waals attraction and surface charges, dominate interactions. The inertia of the dispersed phase is small enough such that it exhibits Brownian motion, a random walk driven by momentum imparted by collision with molecules of the suspending medium. Sol can be prepared by two techniques, condensation and dispersion of particles [16]. Condensation proceeds by nucleation growth of particles to the appropriate size, whereas dispersion involves the reduction of large particles down to the colloidal dimensions. The size and properties of the resulting particles depend on the relative rates of these two processes. Sol formation is favored when the rate of nucleation is high and the rate of crystal growth is low. Depending on the degree of crosslinking and the growth process by which they are formed, the inorganic clusters can be either colloidal or polymeric in nature and can range from 10 to $200 \AA$ in diameter. Generation of inorganic sols also requires controlled conditions, such that the resulting sol is stable with respect to agglomeration and precipitation. Several factors, such as polarity of the solvent, ionic strength of the reaction medium, and temperature, can be used to manipulate the formation of the sol.

Gelation is the process whereby a free flowing sol is converted into a 3D solid network enclosing the solvent medium. A gel is a semisolid rich in liquid. It is interesting to note that liquid does not allow the solid network to collapse, and the solid network does not allow the liquid to flow out [17]. The point of gelation is typically identified by an abrupt rise in viscosity and an elastic response to stress. For preparation of aerogels, the gelation is most conveniently induced through a change in the $\mathrm{pH}$ of the reaction solution. The mechanical state of the gel depends very much upon the number of cross-links in the network. It is obvious that the greater the degree of cross-linking, the more rigid the structure formed.

2.2. Chemistry of Sol-Gel Process. There are several parameters which influence the hydrolysis and condensation reactions (sol-gel process), including the activity of the metal alkoxide, the water/alkoxide ratio, solution $\mathrm{pH}$, temperature, nature of the solvent, and additive used. Another consideration is that catalysts are frequently added to control the rate and the extent of hydrolysis and condensation reactions. By varying these processing parameters, materials with different microstructures and surface chemistry can be obtained. Further processing of the "sol" enables the fabrication ceramic materials in different forms. Thin films can be produced on a piece of substrate by spin coating or dip-coating. When the "sol" is cast into a mold, a wet "gel" will form. With further drying and heat-treatment, the "gel" is converted into dense ceramic or glass particles. If the liquid in a wet "gel" is removed under supercritical conditions, a highly porous and extremely low-density material called an "aerogel" is obtained. The evidence of silicate hydrolysis and condensation to form polysilicate gel and particles is seen in many natural systems like opals and agates [18]. The first metal alkoxide was prepared from $\mathrm{SiCl}_{4}$ and alcohol by Ebelmen, who found that the compound gelled on exposure to the atmosphere and $\mathrm{Si}-\left(\mathrm{OC}_{2} \mathrm{H}_{5}\right)_{4}$ can therefore be regarded as the first "Precursor" for glassy materials [19].

2.3. Precursors for Sol-Gel Processing. The precursor is nothing but the starting materials for the sol-gel process.

(1) Precursors should be soluble in the reaction media.

(2) They should be reactive enough to participate in the gel forming process [20].

Some salts, oxides, hydroxides, complexes, alkoxides, acylates, and amines are used as precursors if soluble in proper solvents [21, 22]. Alkoxides are the most common sol-gel precursor, since they are commonly available. Bradley et al. 
have well explained the basic chemistry of the precursor [23]. It is very difficult to predict the type of precursor to be used for a given purpose. The reactivity of precursor does not depend only on its chemical nature but also on the applied reaction condition [24]. Compared to the precursors of other element, the network forming power of $\mathrm{Si}$ is more to build up a gel [18]. That is why other expensive alkoxide precursors can be substituted by cheaper ones like silicon alkoxide such as TEOS, TMOS, and water-soluble precursor such as $\mathrm{Na}_{2} \mathrm{SiO}_{3}$ for sol-gel processing.

Hydrophobic aerogels obtained from the precursor without surface chemical modification are called hydrophobic precursors, and that of hydrophilic are called hydrophilic precursor as provided in Table 1.

\subsection{Reaction Mechanism.}

2.4.1. For Silicon Alkoxide. Silicate gels are synthesized by hydrolyzing monomeric tetrafunctional and trifunctional silicon alkoxide precursors employing a mineral acid (e.g., $\mathrm{HCl}, \mathrm{C}_{2} \mathrm{O}_{4} \mathrm{H}_{4}$ ) or a base (e.g., $\mathrm{NH}_{3}, \mathrm{NH}_{4} \mathrm{OH}$ ) as a catalyst. The following sol-gel reactions occur during silica network formation $[25,26]$.

Hydrolysis.

$$
\begin{gathered}
\mathrm{Si}(\mathrm{OR})_{4}+4 \mathrm{H}_{2} \mathrm{O} \underset{\begin{array}{c}
\text { Esterification } \\
\text { Si }(\mathrm{OH})_{4}+4 \mathrm{ROH} \\
\text { Silicic acid, }
\end{array}}{\stackrel{\text { Hydrolysis }}{\longrightarrow}}
\end{gathered}
$$

where $\mathrm{R}=$ Vinyl, Alkyl, or Aryl groups.

\section{Condensation.}

(a) Water condensation:

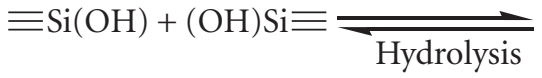

$$
\begin{aligned}
& \equiv \mathrm{Si}-\mathrm{O}-\mathrm{Si} \equiv+\mathrm{H}_{2} \mathrm{O}
\end{aligned}
$$

(b) Alcohol condensation:

$$
\begin{aligned}
& \equiv \mathrm{Si}(\mathrm{OH})+\left(\mathrm{OCH}_{3}\right) \mathrm{Si} \equiv \underset{ }{\stackrel{\text { Alcoholysis }}{\rightleftharpoons}} \\
& \equiv \mathrm{Si}-\mathrm{O}-\mathrm{Si} \equiv+\mathrm{CH}_{3} \mathrm{OH} .
\end{aligned}
$$

2.4.2. Water Soluble Silicates and Minerals. Sodium silicate $\left(\mathrm{Na}_{2} \mathrm{SiO}_{3}\right)$ has been and probably will always be the cheapest source of relatively pure silicic acid from which silica gel can be made. Sodium silicate reacts with water to give silicic acid and then the silicic acid polymerizes and forms silica gel as shown in the following reactions:

$$
\mathrm{Na}_{2} \mathrm{SiO}_{3}+\mathrm{H}_{2} \mathrm{O}+2 \mathrm{HCl} \longrightarrow \mathrm{Si}(\mathrm{OH})_{4}+2 \mathrm{NaCl} .
$$

The silicic acid condenses to form small silica particles, chains and consequently forms a network resulting in a silica gel as shown below.

$$
\begin{aligned}
& n\left[\mathrm{Si}(\mathrm{OH})_{4}+(\mathrm{OH})_{4} \mathrm{Si}\right] \longrightarrow
\end{aligned}
$$

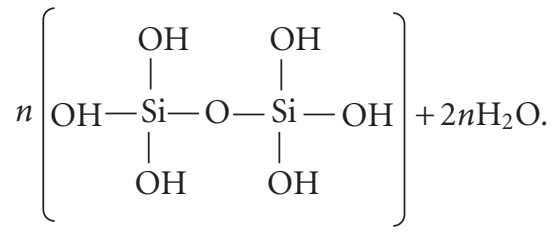

Further, there are some reports available on preparation of silica gels using aluminosilicates, calcium silicates, wollestonite, and so forth [27-29].

It is evident from reactions (2)-(5) that the structure of sol-gel glasses evolves sequentially as the product of successive hydrolysis and condensation reactions (and reverse reactions, i.e., esterification and alcoholic or hydrolytic depolymerization) [15]. Thus, knowledge of mechanisms and kinetics of these reactions will provide insight into the gels and gel-derived glasses.

The hydrolysis reaction is catalyzed by the addition of an acid or a base [29]. In fact, the final form of hydrolyzed silica depends on the $\mathrm{pH}$ of the solution. At low $\mathrm{pH}$ levels (highly acidic), the silica particle tends to form a linear chain with low crosslink density. This leads to a soft gel, which is reversible and can be redispersed in solution. As the $\mathrm{pH}$ value increases, the number of cross-links between the polymer chains also increases. At high $\mathrm{pH}$ (highly basic), the polymers become more branched and the number of cross-links increases. At low $\mathrm{pH}$, hydrolysis occurs by electrophilic attack on the oxygen atom of the alkoxide group, whereas at higher $\mathrm{pH}$, hydrolysis and polymerization occur by nucleophilic attack on the $\mathrm{Si}$ ion $\left(\mathrm{Si}^{4+}\right)$. Atoms, ions, or groups which have a strong affinity for electrons are termed electrophiles, while positive ions are termed nucleophiles. In general, all electrophiles are oxidizing agents and all nucleophiles are reducing agents.

It is generally found that the process of gelation proceeds with smaller segments dissolving and redepositing onto the larger chains so that the smaller molecules decrease in number but assist the larger molecules to grow until they form fractal aggregates. This process is called Ostwald ripening [30].

2.4.3. Hydrophilic and Hydrophobic Surfaces. The names hydrophobic and hydrophilic arise from the combination of "hydro", meaning water in Greek means, "phobos" meaning "hating" in Greek, and "philic" meaning "loving" in Greek. These terms describe the apparent repulsion and attraction between water and surfaces. As shown in Figure 1, hydrophilicity or hydrophobicity is distinguished from the value of contact angle: smaller or larger than $90^{\circ}$. 
TABle 1: Precursors for silica aerogel synthesis.

\begin{tabular}{lc}
\hline Hydrophilic precursors & Hydrophobic precursors \\
\hline Tetramethoxysilane $(\mathrm{TMOS})$ & Methyltrimethoxysilane \\
Tetraethoxysilane $(\mathrm{TEOS})$ & Methyltriethoxysilane $($ MTES) \\
Sodium Silicate $\left(\mathrm{Na}_{2} \mathrm{SiO}_{3}\right)$ & (i) Aerogels-opaque. \\
(i) Aerogels- high optical transmission $(>90 \%)$. & (ii) Density $>0.1 \mathrm{~g} / \mathrm{cm}^{3}$. \\
(ii) Density $<0.1 \mathrm{~g} / \mathrm{cm}^{3}$. & (iii) Soft and flexible. \\
(iii) Hard \& Brittle. & \\
\hline
\end{tabular}

When the surface energy of the solid is low it repels the water from its surface and vice versa, showing hydrophobicity or hydrophilicity. Currently, hydrophobic surfaces are used in industry for a variety of applications including hydrophobic coatings for rust prevention, oil removal from water, management of oil spills, and chemical separation processes to separate non-polar and polar compounds.

For synthesis of hydrophobic and hydrophilic aerogels, two main steps are involved: (a) synthesis of alcogel by sol-gel process and (b) drying of the alcogel by various techniques.

\section{Drying of Alcogel}

After gel formation by hydrolysis and condensation reactions, an Si-O-Si network is formed. The term aging refers to the strengthening of the gel network; it may involve further condensation, dissolution, and reprecipitation of the sol particles or phase transformations within the solid or liquid phases. This results in a porous solid in which the solvent is trapped. The process of removing the majority solvent from the gel (which in the case of an alkoxide-derived gel is mainly alcohol and water) is called drying. During the drying process, cracking of the gel network occurs due to capillary forces that set up in the fine pores by the liquidvapour interfaces. The Laplace equation applies in this case, as the smaller the capillary radius is, the higher the liquid will rise, or the higher the hydrostatic pressure will be. Since this surface energy is responsible for the rise of a column of liquid in a capillary, the magnitude of an interfacial pressure within a capillary can be calculated by balancing the static forces that is,

$$
\begin{aligned}
& 2 \pi r \gamma \cos \theta=\pi r^{2} h \rho g, \\
& h \rho g=p_{r}=2 \gamma \cos \frac{\theta}{r} .
\end{aligned}
$$

The diameters of the pores in the gel are on the order of nanometers, so that the gel liquid must exert highhydrostatic pressure. The meniscus in the pores and the surface tension forces try to pull the particles together as the liquid in the pores evaporates. These forces can act in such a way that they try to collapse the pores, and hence the structure. Thus, the gels with very fine pores have a tendency to crack and shrink during drying. To avoid this drying stress, Kistler described the first synthesis of an aerogel by supercritical drying in the early 1930s [31], and various

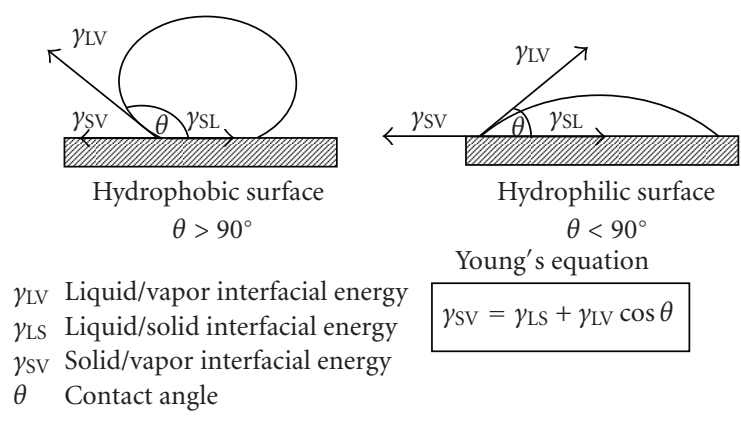

FIGURE 1: Hydrophobic and hydrophilic surfaces.

aerogel synthesis processes have been reported since. In the 1970 s, silica aerogels were synthesized by high temperature supercritical drying of a wet gel produced by the hydrolysis of TMOS in methanol [32]. In the 1980s, researchers gained a new understanding of the potential of aerogels, and TEOSbased silica aerogels, whose synthesis was less expensive and used fewer toxic sources compared to TMOS-based aerogels, were developed. The low-temperature supercritical drying technique, which uses liquid carbon dioxide, was introduced at the same time [33].

3.1. Supercritical Drying of the Alcogel. In supercritical drying methods, gels are dried at a critical point to eliminate the capillary forces, as described below.

As soon as the liquid begins to evaporate from the gel, surface tension creates concave menisci in the pores of the gel. As the evaporation of the liquid continues, compressive forces build up around the perimeter of the pore and it contracts. Eventually, surface tension causes the collapse of the gel body [34]. In order to prevent the surface tension from building up, the gel is dried supercritically in an autoclave, as shown in Figure 2. When the temperature and the pressure in the autoclave are increased above a critical point (for methanol the critical temperature and the critical pressure values are $243^{\circ} \mathrm{C}$ and $7.9 \mathrm{MPa}$, resp.), the liquid is transformed into a "supercritical" fluid in which every molecule can move about freely and the surface tension ceases. Without surface tension, menisci do not form. The vapours are then slowly released from the autoclave, until the pressure in the autoclave reaches atmospheric pressure. Finally, the autoclave is flushed with dry nitrogen ( $\sim 3$ bar) in order to remove the trapped solvent molecules from the 


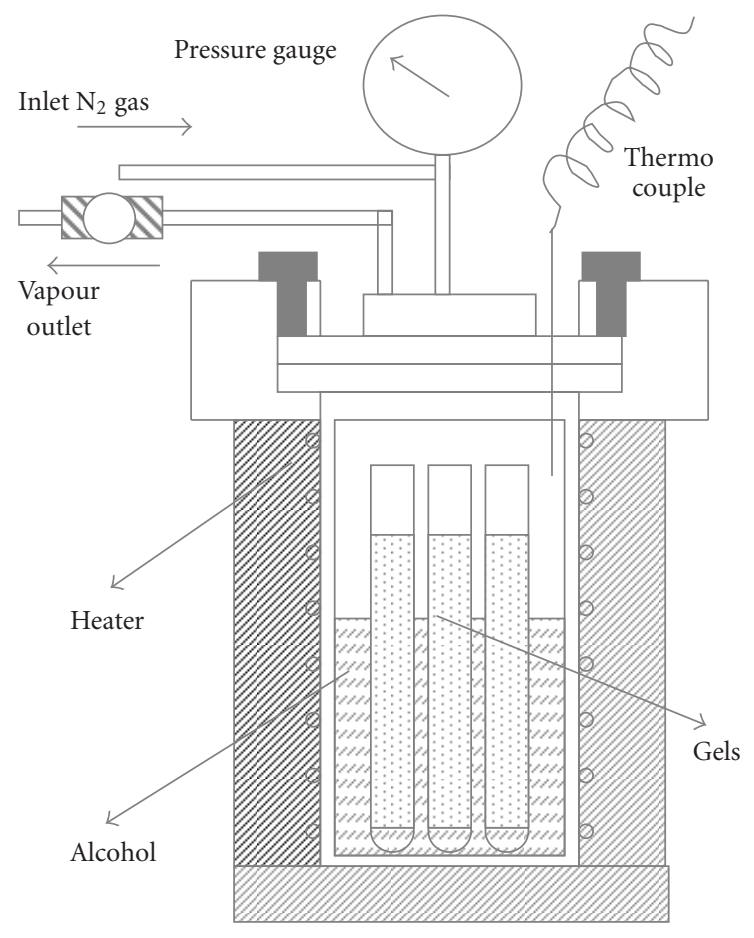

FIGURE 2: Schematic representation of supercritical drying autoclave.

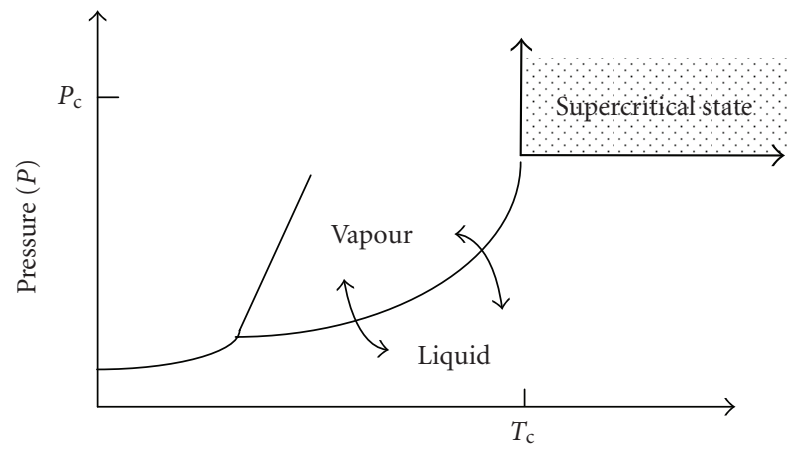

Temperature (T)

Figure 3: Pressure-temperature correlation for solid- liquid-vapour phase equilibrium phase diagram.

dried gel. This method of drying of the alcogels is referred to as "supercritical drying". Figure 3 shows the pressuretemperature cycles followed during the supercritical drying of the alcogels.

3.2. Ambient Pressure Drying and Surface Chemical Modification. Traditionally, silica aerogels have been synthesized using supercritical drying methods, but this has certain limitations in terms of its cost efficiency, process continuity, and safety because a high-temperature and pressure are needed to approach the critical point. If liquid carbon dioxide were used as a solvent in the low-temperature supercritical drying process, the chemical durability of the aerogels in the atmosphere would be gradually decreased, since the aerogel particles are hydrophilic. To overcome these problems, Brinker introduced a commercially attractive ambient pressure drying method for the production of silica aerogel [35]. In this process, the surface of the wet gel is chemically modified by substituting hydrophobic functional groups by replacement of $\mathrm{H}$ from hydroxyl groups followed by ambient pressure drying. Surface silanol groups ( $\mathrm{Si}-\mathrm{OH})$ on the adjacent silica cluster undergo condensation reactions resulting in an irreversible shrinkage of the gel network during drying, as shown in Figure 4. This process can create surfaces with extremely low energies, which dramatically reduce surface tension. Therefore, it is necessary to modify alcogel surfaces with appropriate modifying agents, so that the surface of the aerogel is rendered hydrophobic.

There are several substances capable of altering the wetting properties of the surface, that is, hydrophobic reagents. These include methyltrimethoxysilane (MTMS), hexamethyldisilazane (HMDZ), dimethylchlorosilane (DMCS), dimethyldichlorosilane (DMDC), trimethylchlorosilane (TMCS), trimethylethoxysilane (TMES), and hexadecyltrimethoxysilane (HDTMS). Surface modification of the gels through the replacement of $\mathrm{H}$ from $\mathrm{Si}-\mathrm{OH}$ by non-polar alkyl or aryl groups is a crucial step in the ambient pressure drying method. That prevents condensation reactions of silica clusters, and, by extension, prevents shrinkage of the gel during drying. Since ambient pressure drying can reduce the production cost of the aerogels, their importance has changed from an area of purely scientific interest in to one of practical usage.

3.3. Freeze Drying. Another possibility to avoid phase boundaries between the liquid and the gas phase during drying is freeze drying. The pore liquid is frozen and then sublimed in vacuo. There were some attempts to use this method for the production of aerogels [36-38]. However, the aging period must be extended to stabilize the gel network, the solvent must be replaced by one with a low expansion coefficient and a high sublimation pressure, and low freezing temperatures are attained by addition of salts. Another disadvantage is that the network may be destroyed by crystallization of the solvent in the pores. Cryogels are therefore only obtained as powders.

\section{Aerogel Properties and Applications}

Aerogels have some unique properties which makes them attractive in science and technology, as given in Table 2. Due to these unique properties, aerogels are used for various applications as mentioned in Table 3 , and some recent applications are as briefly discussed below.

4.1. Aerogel as a Composite. As silicon alkoxide precursor is reactive enough to form gel networks with other metal oxides, several studies were carried out to synthesized silica aerogel composites for various applications. Structural and magnetic properties of silica aerogel-iron oxide nanocomposites were studied by Casas et al. [39, 40]. Figure 5 shows a silica-titania aerogel composite synthesized via 

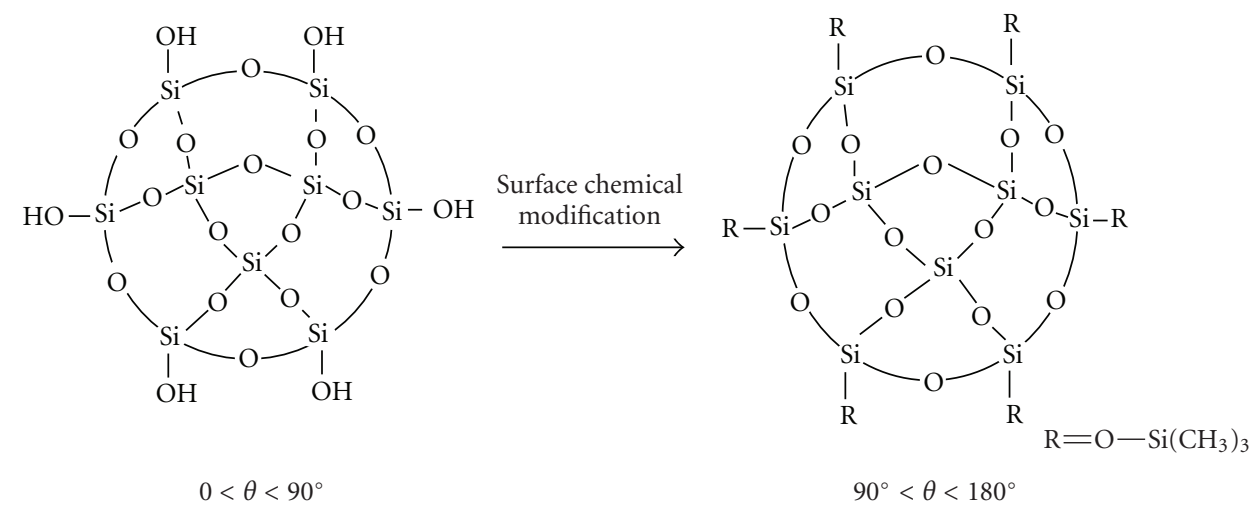

FIgURE 4: Surface chemical modification of the gel.

TABLE 2: Typical properties of silica aerogels.

\begin{tabular}{lc}
\hline Property & Value \\
\hline Apparent density & $0.03-0.35 \mathrm{~g} / \mathrm{cm}^{3}$ \\
Internal surface area & $600-1000 \mathrm{~m}^{2} / \mathrm{g}$ \\
$\%$ solids & $0.13-15 \%$ \\
Mean pore diameter & $\sim 20 \mathrm{~nm}$ \\
Primary particle diameter & $2-5 \mathrm{~nm}$ \\
Refractive index & $1.0-1.08$ \\
Coefficient of thermal expansion & $2.0-4.0 \times 10^{-6}$ \\
Dielectric constant & $\sim 1.1$ \\
Sound velocity & $100 \mathrm{~m} / \mathrm{s}$ \\
\hline
\end{tabular}

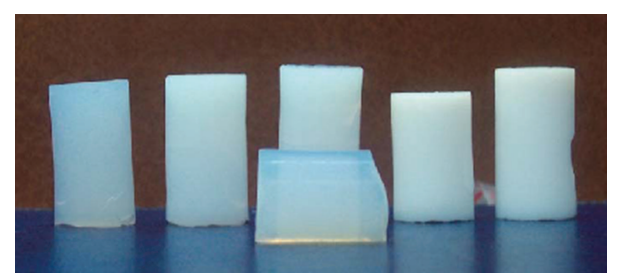

Figure 5: Photographs of silica-titania aerogels (from left $5 \mathrm{wt} \%$ and Four $10 \mathrm{wt} \%$. In front $2 \mathrm{wt} \%$ samples).

ambient pressure drying. There are several reports which describe synthesis of silica-titania[41], silica-carbon, silica and alumina microfibers, [42] or activated carbon powder [43] composite aerogels.

4.2. Aerogel as an Absorbent. Synthesis of flexible and super hydrophobic aerogels and their use in absorption of organic solvents and oils were studied by A. Venkateshwara Rao et al. $[44,45]$. They investigated the absorption and desorption capacity of super hydrophobic silica aerogels using eleven solvents and three oils. Figure 6 shows various stages of absorption and desorption of organic liquids from the aerogel.

The mass $(\mathrm{m})$ of a liquid that rises into the capillaries (aerogel pores) is given by the following formula:

$$
2 \pi \mathrm{r} \gamma \cos \theta=\mathrm{mg} .
$$

For liquids that completely wet the surface, the contact angle $\theta$ is zero and for such surfaces:

$$
2 \pi \mathrm{r} \gamma=\mathrm{mg}
$$

or

$$
\gamma=\mathrm{km},
$$

or

$$
\gamma=\mathrm{kV} \rho
$$

or

$$
\frac{\gamma}{\rho}=\mathrm{kV},
$$

where $r$ is the radius of the aerogel pores, $V$ is the volume of the liquid absorbed, $\rho$ is the density of the liquid and $\mathrm{k}=$ $\mathrm{g} / 2 \pi \mathrm{r}$ is a constant for the given aerogel sample. Therefore, it follows from (10) that the mass of the liquid absorbed increases linearly with an increase in the surface tension of the liquid. Elastic superhydrophobic MTMS aerogels were found to be effective and efficient absorbents of oils and organic liquids.

4.3. Aerogel as a Sensor. Aerogels have high overall porosity, good pore accessibility, and high surface active sites. They are therefore potential candidates for use as sensors. A study by Wang et al. [46] on silica nanoparticle aerogel thin films showed that their electrical resistance markedly decreases with increasing humidity. They are highly sensitive to $40 \%$ $\mathrm{RH}$ and greater and operate with a $3.3 \%$ hysteresis, which is attributed to their pore structure. Xerogels of the same material, on the other hand, show very low sensitivity. Surface modified aerogels are less affected by humidity as compared to hydrophilic aerogels and can be used as anticorrosive, hydrophobic agents, as shown in Figure 7 [47].

Wub and Chen-yang [48] studied aerogels for biosensor applications. In this study, mesoporous aerogels were prepared at room temperature by sol-gel polymerization with an ionic liquid as the solvent and pore-forming agent. The asprepared aerogel was characterized by different instruments 


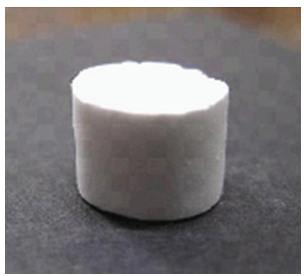

(a) Before absorption

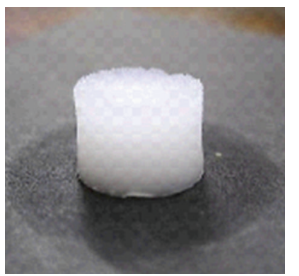

(b) Immediately after absorption, $t=0 \mathrm{~min}$

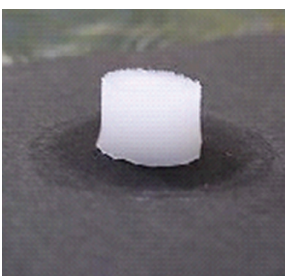

(c) At $t=20 \mathrm{~min}$

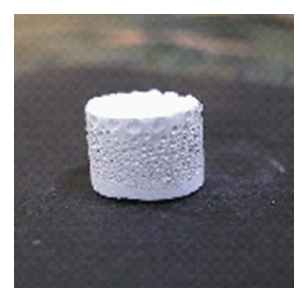

(d) At $t=30 \mathrm{~min}$.

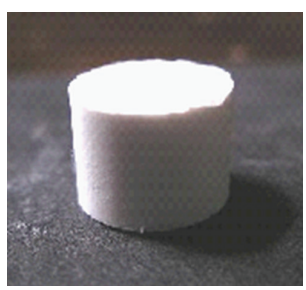

(e) After desorption, $t=$ $40 \mathrm{~min}$.

FIGURE 6: Picture showing various stages of absorption and desorption of organic liquid from the aerogel.

and was found to have high porosity and large internal networking surface area. The as-prepared aerogel was further arrayed onto slides and successfully recognized a short human gene ATP5O by an immobilized oligonucleotide probe on the aerogel surface, as shown in Figure 8. The large capturing capacity of the porous structure was also demonstrated by comparing with a planar surface at high target concentrations. The results indicate that the asprepared aerogel can function as a recognition substrate for nucleotide acids. This report proposes a preparation technique to synthesize mesoporous aerogel using the solgel process and utilize the aerogel's high surface area and large internal porous volume for molecular recognition of nucleotide acids.

4.4. Aerogel as Material with Low-Dielectric Constant. $\mathrm{SiO}_{2}$ aerogel thin films have received a significant attention in IC applications because of their unique properties such as their ultralow dielectric constants, high porosity, and high thermal stability. Park et al. investigated silica aerogel thin films for interlayer dielectrics, and the dielectric constant was measured to be approximately 1.9 [48-52]. They produced ultra low dielectric constant aerogel films for intermetal dielectric (IMD) materials. The $\mathrm{SiO}_{2}$ aerogel films having a thickness of $9500 \AA$, a high porosity of $79.5 \%$, and a low dielectric constant of 2.0 were obtained by a new ambient drying process using $n$-heptane as a drying solvent.

4.5. Aerogel as Catalysts. The high surface area of aerogels leads to many applications, such as a chemical absorber for cleaning up spills. This feature also gives it a great potential as a catalyst or a catalyst carrier. Aerogels aid in heterogeneous catalysis, when the reactants are either in gas or liquid phase. They are characterized by very high surface area per unit mass, high porosity which makes them a very attractive option for catalysis. Some of the reactions catalyzed by aerogels are listed below.

\section{Some Examples of Aerogels in Catalysis.}

(1) Synthesis of nitrile from hydrocarbons using nitric oxide (NO) [53].

(2) Isobutene can be converted into methacrylonitrile by reacting it with $\mathrm{NO}$ on zinc oxide aerogel [54].

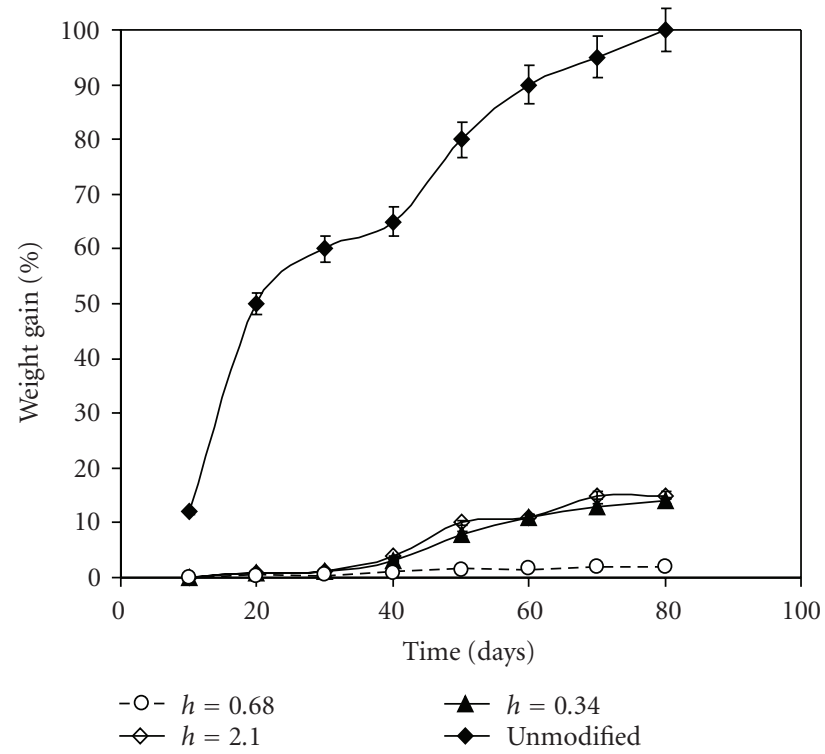

FIGURE 7: Effect of humidity on surface modified and unmodified aerogel.

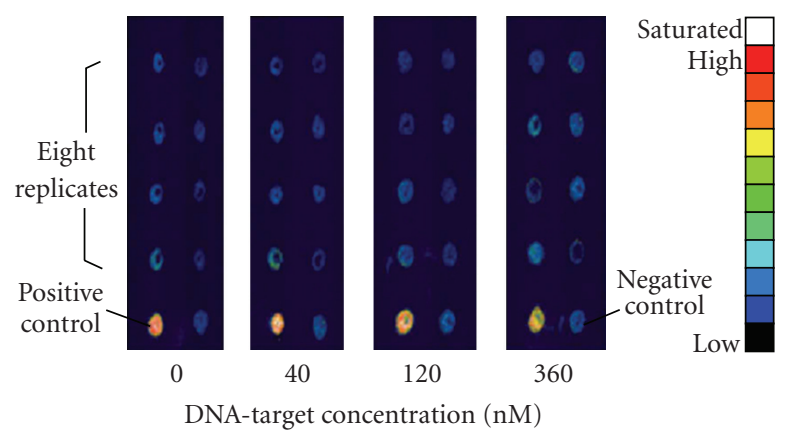

FIGURE 8: Nonspecific molecular recognition test on the aerogel biochips. Human gene PSMA5 (432 b) served as the target and was tested by 10 lMATP5Oc probe immobilized on the aerogel surface.

(3) Synthesis of methanol from CO using copper zirconia aerogel [55].

4.6. Aerogel as a Storage Media. The high porosity and very large surface area of silica aerogels can also be utilized for 
TABLE 3: Applications of the aerogel perspective to properties.

\begin{tabular}{|c|c|c|}
\hline Property & Feature & Application \\
\hline \multirow[t]{4}{*}{ Thermal conductivity } & (i) Best insulating solid & (i) Building construction and appliance insulation \\
\hline & (ii) Transparent & (ii) Storage media \\
\hline & (iii) Withstand high temperature & (iii) Automobiles, Space vehicles \\
\hline & (iv) Light weight & (iv) Solar devices, solar ponds \\
\hline \multirow[t]{8}{*}{ Density/porosity } & (i) Lightest synthetic solid & (i) Catalysis \\
\hline & (ii) High surface area & (ii) Sensor \\
\hline & (iii) Multiple compositions & (iii) Fuel storage \\
\hline & & (iv) Ion exchange \\
\hline & & (v) Filters for pollutants gaseous \\
\hline & & (vi) Targets for ICF \\
\hline & & (vii) Pigment carriers \\
\hline & & (viii) Template \\
\hline \multirow[t]{3}{*}{ Optical } & (i) Transparent & (i) Light weight optics \\
\hline & (ii) Low refractive index & (ii) Cherenkov detectors \\
\hline & (iii) Multiple composition & (iii) Light guides \\
\hline \multirow[t]{2}{*}{ Acoustic } & (i) Low speed of sound & (i) Sound proof rooms \\
\hline & & (ii) Acoustic impedance matching in ultrasonic distance sensors \\
\hline \multirow[t]{2}{*}{ Mechanical } & (i) Elastic & (i) Energy absorber \\
\hline & (ii) Light weight & (ii) Hypervelocity particle trap \\
\hline \multirow[t]{3}{*}{ Electrical } & (i) Lowest dielectric constant & (i) Dielectrics for ICs \\
\hline & (ii) High dielectric strength & (ii) Spacers for vacuum electrodes \\
\hline & (iii) High surface area & (iii) Capacitors \\
\hline
\end{tabular}

applications as gas filters, absorbing media for desiccation and waste containment, encapsulation media, and hydrogen fuel storage [54]. Partially sintered aerogels can resist the tensions of a gas/liquid interface because their texture is strengthened during sintering. They can therefore be used for the storage, thickening, or transport of liquids, for example, rocket fuels. In the latter case, the low weight of aerogels is particularly advantageous. Aerogel can be used in drug delivery systems due to their biocompatibility [56-58]. Carbon aerogels are used in the construction of small electrochemical double-layer supercapacitors. Due to the high surface area of the aerogel, these capacitors can be $1 / 2000$ th to $1 / 5000$ th the size of similarly rated electrolytic capacitors [59]. Aerogel supercapacitors can have very low impedance compared to normal supercapacitors and can absorb or produce very high-peak currents. At present, such capacitors are polarity-sensitive and need to be wired in series if a working voltage of greater than about $2.75 \mathrm{~V}$ is needed.

4.7. Aerogel as a Template [60]. Hupp et al. used silica aerogel films for dye sensitized solar cells. High surface area mesoporous aerogel films were prepared on conductive glass substrates. Atomic layer deposition was employed to coat the aerogel template conformally with various thicknesses of $\mathrm{TiO}_{2}$ with subnanometer precision. The $\mathrm{TiO}_{2}$-coated aerogel membranes were incorporated as photoanodes in dye-sensitized solar cells. The charge diffusion length was found to increase with increasing thickness of $\mathrm{TiO}_{2}$ leading to increasing current and efficiency.

4.8. Aerogel as a Thermal Insulator. Apart from high porosity and low-density one of the most fascinating properties of aerogels is their very low thermal conductivity. Aerogels possess a very small thermal conductivity, $\sim 1-10 \%$ that of a solid, additionally, they consist of very small particles linked in a 3-dimensional network with many "dead-ends". Therefore, thermal transport through the solid portion of an aerogel occurs through a very tortuous path and is not particularly effective. The space not occupied by solids in an aerogel is normally filled with air (or another gas) unless the material is sealed under vacuum. These gases can also transport thermal energy through the aerogel. The pores of aerogel are open and allow the passage of gas (albeit with difficulty) through the material.

4.8.1. In Housing, Refrigerators, Skylights, and Windows. Silica aerogels can be synthesized using low cost precursors at ambient pressure which makes aerogels suitable for commercialization. Aerogels transmit heat only one hundredth as well as normal density glass. The first residential use of aerogels is as an insulator in the Georgia Institute of Technology's Solar Decathlon House, where it is used as an insulator in the semitransparent roof. Aerogels are a more efficient, low-density form of insulation than the polyurethane foam currently used to insulate refrigerators, refrigerated vehicles, 
and containers. Foams are blown into refrigerator walls by chlorofluorocarbon (CFC) propellants, the chemical that is the chief cause of the depletion of the earth's stratospheric ozone layer. Replacing chlorofluorocarbon-propelled refrigerant foams with aerogels could help eliminate this problem.

4.8.2. In Clothing, Apparel, and Blankets. Commercial manufacture of aerogel "blankets" began around the year 2000. An aerogel blanket is a composite of silica aerogel and fibrous reinforcement that turns the brittle aerogel into a durable and flexible material. The mechanical and thermal properties of the product may be varied based upon the choice of reinforcing fibers, the aerogel matrix, and pacification additives included in the composite. Aspen Aerogels Inc. of Marlborough, Massachusetts has produced a Spaceloft product, an inexpensive and flexible blanket that incorporates a thin layer of aerogel embedded directly into the fabric. Another type of aerogels is organic, which is made of carbon and hydrogen atoms. Mount Everest climbers have used Aerogel insoles, as well as sleeping bags lined with the material.

4.8.3. In Space. NASA used aerogels to trap space dust particles aboard the Stardust spacecraft. The particles vaporize on impact with solids and pass through gases, but can be trapped in aerogels. NASA also used aerogel for thermal insulation of the Mars Rover and space suits [61, 62]. The US Navy is evaluating aerogel undergarments as passive thermal protection for divers [63].

\section{Future Perspectives}

The unique optical, thermal, acoustic, and mechanical properties of aerogels originate from the combination of a solid network and nanosized pores filled with air. The choice of the precursors and the optimization of sol-gel parameters determine the physical properties of the final aerogel product. Drying of the alcogels is carried out with supercritical or ambient pressure drying methods, depending upon the commercial aerogel application. An extensive chemical modification provides a new space for studying the property of aerogels. Ambient pressure drying techniques will probably make industrial preparation much cheaper and will thus make aerogels more competitive. The most important area for the application of aerogels is in all kinds of thermal insulation. Also, $\mathrm{SiO}_{2}$ aerogels have some physical and ecological advantages (nontoxic, nonflammable, easy to dispose of) compared with most of other materials in the market.

\section{Acknowledgments}

This work was supported by DAPA and ADD. One of the authors thanks the Brain Korea program Phase-II for providing a BK-21 postdoctorial fellowship.

\section{References}

[1] L. W. Hrubesh, "Aerogels: the world's lightest solids," Chemistry and Industry, no. 24, pp. 824-827, 1990.

[2] G. C. Bond and S. Flamerz, "Structure and reactivity of titania-supported oxides. Part 3: reaction of isopropanol over vanadia-titania catalysts," Applied Catalysis, vol. 33, no. 1, pp. 219-230, 1987.

[3] J. Fricke and A. Emmerling, "Aerogels, preparation, properties, applications," in Structure and Bonding 77: Chemistry, Spectroscopy and Applications of Sol-Gel Glasses, pp. 37-87, Springer, Berlin, Germany, 1992.

[4] C. A. M. Mulder and J. G. Van Lierop, "Preparation, densification and characterization of autoclave dried $\mathrm{SiO}_{2}$ gels," in Aerogels, J. Fricke, Ed., pp. 68-75, Springer, Berlin, Germany, 1986.

[5] Y. Xu, G. Huang, and Y. He, "Sol-gel preparation of $\mathrm{Ba}_{6-3 \mathrm{x}} \mathrm{Sm}_{8+2 \mathrm{x}} \mathrm{Ti}_{18} \mathrm{O}_{54}$ microwave dielectric ceramics," Ceramics International, vol. 31, no. 1, pp. 21-25, 2005.

[6] S. Tursiloadi, H. Imai, and H. Hirashima, "Preparation and characterization of mesoporous titania-alumina ceramic by modified sol-gel method," Journal of Non-Crystalline Solids, vol. 350, pp. 271-276, 2004.

[7] R. Jain, V. Gupta, A. Mansingh, and K. Sreenivas, "Ferroelectric and piezoelectric properties of non-stoichiometric $\mathrm{Sr}_{1-\mathrm{x}} \mathrm{Bi}_{2+2 \mathrm{x} / 3} \mathrm{Ta}_{2} \mathrm{O}_{9}$ ceramics prepared from sol-gel derived powders," Materials Science and Engineering B, vol. 112, no. 1, pp. 54-58, 2004.

[8] K. Li, J.-H. Li, and H. L. W. Chan, "Fabrication of Sm- and Mn-doped lead titanate ceramic powder and ceramics by solgel methods," Materials Chemistry and Physics, vol. 86, no. 1, pp. 83-87, 2004.

[9] P. Escribano, M. Marchal, M. Luisa Sanjuán, P. AlonsoGutiérrez, B. Julián, and E. Cordoncillo, "Low-temperature synthesis of $\mathrm{SrAl}_{2} \mathrm{O}_{4}$ by a modified sol-gel route: XRD and Raman characterization," Journal of Solid State Chemistry, vol. 178, no. 6, pp. 1978-1987, 2005.

[10] P. A. S. Jorge, P. Caldas, C. C. Rosa, A. G. Oliva, and J. L. Santos, "Optical fiber probes for fluorescence based oxygen sensing," Sensors and Actuators B, vol. 103, no. 1-2, pp. 290-299, 2004.

[11] H. Matsuda, N. Kobayashi, T. Kobayashi, K. Miyazawa, and M. Kuwabara, "Room-temperature synthesis of crystalline barium titanate thin films by high-concentration sol-gel method," Journal of Non-Crystalline Solids, vol. 271, no. 1, pp. 162-166, 2000.

[12] D. Torikai, C. K. Suzuki, H. Shimizu et al., "Comparison of high-purity $\mathrm{H}_{2} / \mathrm{O}_{2}$ and $\mathrm{LPG} / \mathrm{O}_{2}$ flame-fused silica glasses from sol-gel silica powder," Journal of Non-Crystalline Solids, vol. 179, pp. 328-334, 1994.

[13] H. C. Hamker, "A general theory of lyophobic colloids I," Journal of Royal Netherlands Chemical Society, vol. 55, p. 1015, 1936.

[14] J. Lyklema, S. Levine, A. L. Smith et al., "General discussion," Discussions of the Faraday Society, vol. 52, pp. 312-323, 1971.

[15] F. Schuth, K. S. W. Sing, and J. Weitkamp, Handbook of Porous Solids, vol. 3, Wiley-VCH, Weinheim, Germany, 2002.

[16] P. J. Flory, Principal of Polymer Chemistry, chapter 4, Cornell University Press, Ithaca, NY, USA, 1953.

[17] C. J. Brinker, "Hydrolysis and condensation of silicates: effects on structure," Journal of Non-Crystalline Solids, vol. 100, no. 1-3, pp. 31-50, 1988. 
[18] E. Tkalcec, R. Nass, J. Schmauch et al., "Crystallization kinetics of mullite from single-phase gel determined by isothermal differential scanning calorimetry," Journal of Non-Crystalline Solids, vol. 223, no. 1-2, pp. 57-72, 1998.

[19] A. C. Pierre and G. M. Pajonk, "Chemistry of aerogels and their applications," Chemical Reviews, vol. 102, no. 11, pp. 4243-4266, 2002.

[20] J. Livage, Ph. Barboux, E. Tronic, and J. P. Jolivet, Science of Ceramic Processing, H. Hench and D. Ulrich, Eds., Wiely, New York, NY, USA, 1986.

[21] W. C. LaCourse and S. Kim, "Sol-gel processes for fibers and films of multicomponent materials," Science of Ceramic Processing, vol. 8, no. 9-10, pp. 1128-1134, 1987.

[22] D. C. Bradley, R. C. Mehrotra, and D. P. Gaur, Metal Alkoxides, Academic Press, New York, NY, USA, 1978.

[23] H. Schmidt, "Chemistry of material preparation by sol-gel process," Journal of Non-Crystalline Solids, vol. 100, pp. 51-64, 1988.

[24] F. D. Ovcharenako, Y. I. Tarasevich, et al., Kolloidnyj Zhurnal, vol. 34, p. 412, 1972.

[25] C. J. Brinker and G. W. Scherer, Sol-Gel Science: The Physics and Chemistry of Sol-Gel Processing, Academic Press, San Diego, Calif, USA, 1990.

[26] J. L. Gurav, D. Y. Nadargi, and A. V. Rao, "Effect of mixed Catalysts system on TEOS-based silica aerogels dried at ambient pressure," Applied Surface Science, vol. 255, no. 5, pp. 3019-3027, 2008.

[27] E. M. Flanigen and R. W. Grose, Siliceous Adsorbent Materials And Process For Preparing Same, U.S. Patent no. 3, 494, 874, 1970.

[28] K. J. Murata and W. G. Schlecht, The significance of internal structure in gelatinizing silicate minerals, U.S. Geological Survey Bulletin, vol. 950, no. 25-33, pp. 35-82, 1946.

[29] M. G. Vorankov, V. P. Mileshkevich, and Y. A. Yuzhelevski, The Siloxane Bond, Consultant Bureau, New York, NY, USA, 1978.

[30] W. Z. Ostwald, "Studies on formation and transformation of solid materials," Physical Chemistry, vol. 22, pp. 289-330, 1897.

[31] S. S. Kistler, "Coherent expanded aerogels," Journal of Physical Chemistry, vol. 36, no. 1, pp. 52-64, 1932.

[32] G. A. Nicolaon and S. J. Teichner, "New preparation process for silica xerogels and aerogels, and their textural properties," Bulletin de la Société Chimique de France, vol. 5, pp. 19001906, 1968.

[33] P. H. Tewari, A. J. Hunt, and K. D. Lofftus, "Ambienttemperature supercritical drying of transparent silica aerogels," Materials Letters, vol. 3, no. 9-10, pp. 363-367, 1985.

[34] D. M. Smith, G. W. Scherer, and J. M. Anderson, "Shrinkage during drying of silica gel," Journal of Non-Crystalline Solids, vol. 188, no. 3, pp. 191-206, 1995.

[35] C. J. Brinker and W. Scherer, "Sol-gel sciences," in The Processing and the Chemistry of Sol-Gel Processing, Academic Press, San Diego, Calif, USA, 1990.

[36] B. Mathieu, S. Blacher, R. Pirard, J. P. Pirard, B. Sahouli, and F. Brouers, "Freeze-dried resorcinol-formaldehyde gels," Journal of Non-Crystalline Solids, vol. 212, no. 2-3, pp. 250-261, 1997.

[37] D. Klvana, J. Chaouki, M. Repellin-Lacroix, and G. M. Pajonk, "A new method of preparation of aerogel-like materials using a freeze-drying process," Le Journal de Physique Colloques, vol. 50, no. C4, pp. C4-29-C4-32, 1989.

[38] G. M. Pajonk, "Drying methods preserving the textural properties of gels," Le Journal de Physique Colloques, vol. 50, no. C4, pp. C4-13-C4-22, 1989.
[39] LL. Casas, A. Roig, E. Rodríguez, E. Molins, J. Tejada, and J. Sort, "Silica aerogel-iron oxide nanocomposites: structural and magnetic properties," Journal of Non-Crystalline Solids, vol. 285, no. 1-3, pp. 37-43, 2001.

[40] LL. Casas, A. Roig, E. Molins, J. M. Grenèche, J. Asenjo, and J. Tejada, "Iron oxide nanoparticles hosted in silica aerogels," Applied Physics A, vol. 74, no. 5, pp. 591-597, 2002.

[41] P. Shajesh, S. Smitha, P. R. Aravind, and K. G.K. Warrier, "Effect of 3-glycidoxypropyltrimethoxysilane precursor on the properties of ambient pressure dried silica aerogels," Journal of Sol-Gel Science and Technology, vol. 50, no. 3, pp. 353-358, 2009.

[42] K. E. Parmenter and F. Milstein, "Mechanical properties of silica aerogels," Journal of Non-Crystalline Solids, vol. 223, no. 3, pp. 179-189, 1998.

[43] M. Moner-Girona, E. Martínez, J. Esteve, A. Roig, R. Solanas, and E. Molins, "Micromechanical properties of carbon-silica aerogel composites," Applied Physics A, vol. 74, no. 1, pp. 119$122,2002$.

[44] A. Venkateswara Rao, N. D. Hegde, and H. Hirashima, "Absorption and desorption of organic liquids in elastic superhydrophobic silica aerogels," Journal of Colloid and Interface Science, vol. 305, no. 1, pp. 124-132, 2007.

[45] A. Venkateswara Rao, S. D. Bhagat, H. Hirashima, and G. M. Pajonk, "Synthesis of flexible silica aerogels using methyltrimethoxysilane (MTMS) precursor," Journal of Colloid and Interface Science, vol. 300, no. 1, pp. 279-285, 2006.

[46] C.-T. Wang, C.-L. Wu, I.-C. Chen, and Y.-H. Huang, "Humidity sensors based on silica nanoparticle aerogel thin films," Sensors and Actuators B, vol. 107, no. 1, pp. 402-410, 2005.

[47] J. L. Gurav, A. Venkateswara Rao, and D. Y. Nadargi, "Study of thermal conductivity and effect of humidity on HMDZ modified TEOS based aerogel dried at ambient pressure," Journal of Sol-Gel Science and Technology, vol. 50, no. 3, pp. 275-280, 2009.

[48] Y. K. Li, D.-K. Yang, Y.-C. Chen, H.-J. Su, J.-C. Wu, and Y. W. Chen-Yang, "A novel three-dimensional aerogel biochip for molecular recognition of nucleotide acids," Acta Biomaterialia, vol. 6, no. 4, pp. 1462-1470, 2010.

[49] G. S. Kim, S. H. Hyun, and H. H. Park, "Synthesis of lowdielectric silica aerogel films by ambient drying," Journal of the American Ceramic Society, vol. 84, no. 2, pp. 453-455, 2001.

[50] S.-B. Jung, S.-W. Park, J.-K. Yang, H.-H. Park, and H. Kim, "Application of $\mathrm{SiO}_{2}$ aerogel film for interlayer dielectric on GaAs with a barrier of $\mathrm{Si}_{3} \mathrm{~N}_{4}$," Thin Solid Films, vol. 447-448, pp. 580-585, 2004.

[51] S.-W. Park, S.-B. Jung, M.-G. Kang, H.-H. Park, and H.C. Kim, "Modification of GaAs and copper surface by the formation of $\mathrm{SiO}_{2}$ aerogel film as an interlayer dielectric," Applied Surface Science, vol. 216, no. 1-4, pp. 98-105, 2003.

[52] G. S. Kim, S. H. Hyun, and H. H. Park, "Synthesis of lowdielectric silica aerogel films by ambient drying," Journal of the American Ceramic Society, vol. 84, no. 2, pp. 453-455, 2001.

[53] J. L. Rousset, A. Boukenter, B. Champagnon et al., "Granular structure and fractal domains of silica aerogels," Journal of Physics: Condensed Matter, vol. 2, no. 42, pp. 8445-8455, 1990.

[54] G. M. Pajonk and T. Manzalji, "Synthesis of acrylonitrile from propylene and nitric oxide mixtures on $\mathrm{PbO}_{2}-\mathrm{ZrO}_{2}$ aerogel catalysts," Catalysis Letters, vol. 21, no. 3-4, pp. 361-369, 1993.

[55] A. Sayari, A. Ghorbel, G. M. Pajonk, and S. J. Teichner, "Kinetics of the catalytic transformation of isobutene into methacrylonitrile with NO on supported nickel oxide aerogel," Reaction Kinetics and Catalysis Letters, vol. 15, no. 4, pp. 459$465,1981$. 
[56] H. D. Gesser and P. C. Goswami, "Aerogels and related porous materials," Chemical Reviews, vol. 89, no. 4, pp. 765-788, 1989.

[57] I. Smirnova, S. Suttiruengwong, and W. Arlt, "Feasibility study of hydrophilic and hydrophobic silica aerogels as drug delivery systems," Journal of Non-Crystalline Solids, vol. 350, pp. 54-60, 2004.

[58] I. Smirnova, S. Suttiruengwong, M. Seiler, and W. Arlt, "Dissolution rate enhancement by adsorption of poorly soluble drugs on hydrophilic silica aerogels," Pharmaceutical Development and Technology, vol. 9, no. 4, pp. 443-452, 2004.

[59] M. Juzkow, Aerogel Capacitors Support Pulse, Hold-Up, and Main Power Applications, Cooper Electronic Technologies, PowerStor@ Products, Dublin, Calif, USA, 2002.

[60] T. W. Hamann, A. B. F. Martinson, J. W. Elam, M. J. Pellin, and J. T. Hupp, "Atomic layer deposition of $\mathrm{TiO}_{2}$ on aerogel templates: new photoanodes for dye-sensitized solar cells," Journal of Physical Chemistry C, vol. 112, no. 27, pp. 10303 10307, 2008.

[61] "Preventing heat escape through insulation called "aerogel"', NASA CPL.

[62] "Down-to-Earth Uses for Space Materials," The Aerospace Corporation.

[63] M. L. Nuckols, J. C. Chao, and M. J. Swiergosz, "Manned Evaluation of a Prototype Composite Cold Water Diving Garment Using Liquids and Superinsulation Aerogel Materials," Tech. Rep. NEDU-05-02, United States Navy Experimental Diving Unit, 2005. 

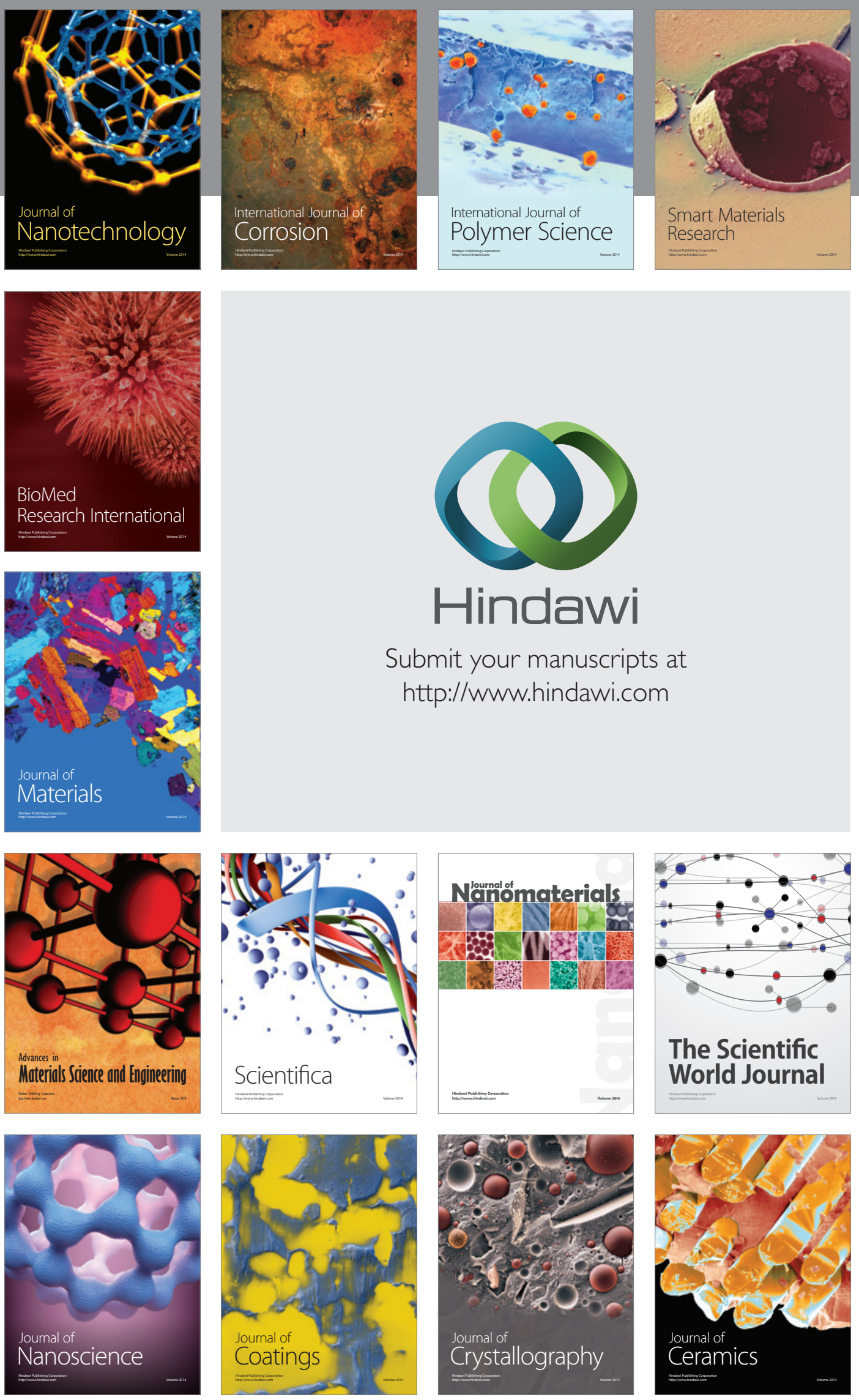

The Scientific World Journal

Submit your manuscripts at

http://www.hindawi.com

\section{World Journal}

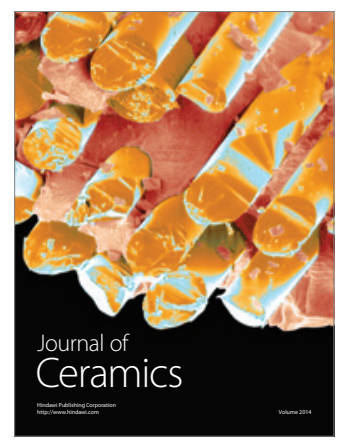

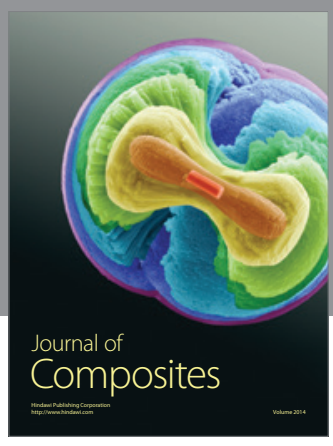
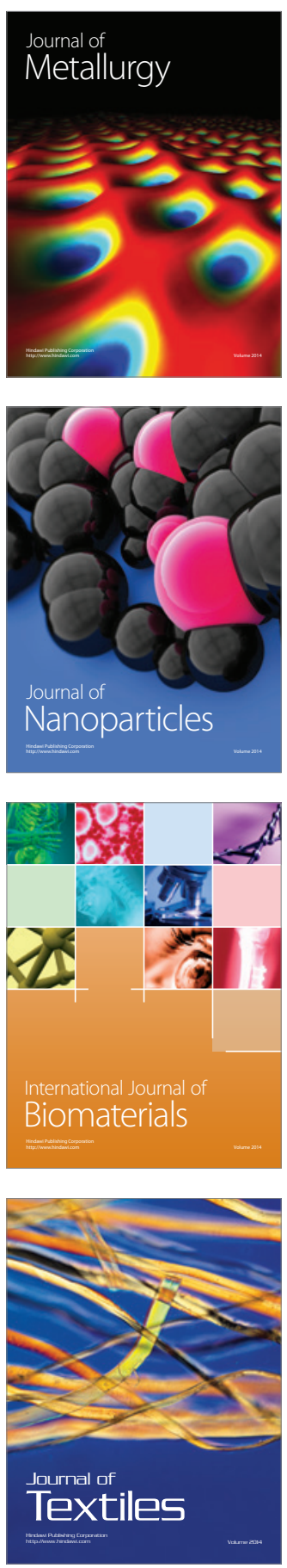